\title{
COMPARAÇÃO ENTRE OS MÉTODOS PSICOFÍSICOS ESCALARES DE ESTIMAÇÃO DE MAGNITUDES E DE ESTIMAÇÃO DE CATEGORIAS DA GRAVIDADE DE QUADROS CLÍNICOS
}

\author{
Ricardo Kamizaki* \\ Fátima Aparecida Emm Faleiros Sousa** \\ José Aparecido da Silva***
}

KAMIZAKI, R.; SOUSA, F.A.E.F.; SILVA, J.A. da. Comparação entre os métodos psicofísicos escalares de estimação de magnitudes e de estimação de categorias da gravidade de quadros clínicos. Rev.latino-am.enfermagem. Ribeirão Preto, v. 8, n. 4, p. 95-102, agosto 2000.

Neste estudo, 15 diagnósticos foram selecionados e avaliados pelo método de estimação de magnitude e de categoria. Os instrumentos foram aplicados em 46 participantes (20 enfermeiros, 16 psicólogos e 10 médicos). Os resultados indicam que a escala psicofísica dos quadros clínicos é um contínuo protético, além da confirmação da validade da lei de Ekman também para contínuos não métricos.

UNITERMOS: psicofisica

O método de estimação de magnitudes foi utilizado com sucesso na mensuração da gravidade de diferentes enfermidades. De fato, WYLER et al. (1968) elaboraram uma lista contendo 126 enfermidades que foi enviada pelo correio a duas amostras distintas: uma não médica e a outra médica. A tarefa dos sujeitos consistia em estimar a magnitude da gravidade das enfermidades assinalando a cada uma delas um número que fosse proporcional ao valor de 500 designado à enfermidade da úlcera péptica. Exemplos de algumas enfermidades foram: constipação intestinal, enxaqueca, diarréia, sinusite, acne, astigmatismo, menopausa, menstruação, eczema, alergia medicamentosa, gonorréia, coma, depressão, epilepsia, derrame cerebral, ataque cardíaco, uremia, câncer e leucemia. As duas amostras foram altamente concordantes em suas estimativas de magnitudes de cada uma dessas enfermidades bem como em suas respectivas ordenações. Os resultados também indicaram que as variáveis idade, sexo, estado civil, etc., afetam numa extensão maior os julgamentos feitos pela amostra não médica do que aqueles feitos pela amostra médica. Combinando as estimativas de magnitudes de ambas as amostras, estas indicaram que a caspa foi a enfermidade com menor estimativa de magnitude, o aborto foi uma enfermidade com estimativa de magnitude mediana e a leucemia foi a enfermidade com maior estimativa de magnitude. O mesmo padrão de resultados foi obtido por WYLER et al. (1971) e VOLICER \& BOHANNON (1975).

Em outro estudo similar, WYLER et al. (1970) replicaram o trabalho original usando uma outra amostra de médicos e analisando as estimativas em função de suas respectivas especialidades médicas. Os resultados mostraram que as estimativas de magnitudes numéricas feitas por médicos de diferentes especialidades não foram significativamente diferentes entre si, exceto apenas para 5 enfermidades, indicando, portanto, que a variável especialidade do respondente não afeta significativamente os resultados.

No experimento de KAMIZAKI et al. (1999) a gravidade de quadros clínicos foi avaliada através do método psicofísico de estimação de magnitudes. Os objetivos deste experimento foram: comparar as escalas derivadas de julgamentos de razão (estimativas de magnitudes) feitos por três diferentes amostras brasileiras e verificar a estabilidade e a concordância das estimativas da gravidade de quadros clínicos julgada no Brasil, E.U.A. e Inglaterra. Para tal foram convidados profissionais atuantes nas áreas de Psicologia, Enfermagem e Medicina. O coeficiente de correlação de

\footnotetext{
* Doutorando, bolsista da FAPESP

** Professor Associado do Departamento de Enfermagem Geral e Especializada da Escola de Enfermagem de Ribeirão Preto da Universidade de São Paulo

*** Professor Titular junto ao Departamento de Psicologia e Educação da Faculdade de Filosofia, Ciências e Letras de Ribeirão Preto da Universidade de São Paulo
} 
Pearson entre os grupos foram de 0,92 o menor e 0,94 o maior. O expoente encontrado entre as amostras brasileira e americana foi de 1,22 e entre as amostras brasileira e inglesa foi de 1,15 .

Desse estudo foram selecionados 15 quadros clínicos (diagnósticos), considerando-se aqueles que apresentavam uma determinada diferença (espaçamento) entre as estimativas de magnitudes para facilitar a discriminação dos quadros clínicos nos julgamentos feitos pelos participantes, ou seja, não foram escolhidos quadros clínicos com estimativas de magnitudes adjacentes muito próximas.

Os objetivos desse Experimento foram: 1) Comparar as escalas derivadas de julgamentos intervalares (estimativas de categorias) com as escalas derivadas de julgamentos de razão (estimativas de magnitudes); 2) Verificar, através da comparação entre as estimativas de magnitudes e as estimativas de categorias, se o contínuo não métrico de gravidade de quadros clínicos tem características protéticas ou metatéticas; 3) Verificar se as ordenações dos graus de gravidade de quadros clínicos derivadas dos dois métodos psicofísicos são similares entre si; 4) Verificar a estabilidade e/ou equivalência das escalas de categorias e de razão através de duas modalidades de respostas diferentes, quais sejam numérica sem limites (estimativas de magnitudes) e numérica com limites (estimativas de categorias) e 5) Verificar a estabilidade e/ou equivalência dessas escalas entre as três amostras.

\section{MÉTODO}

Participantes. Participaram 46 sujeitos, sendo 20 enfermeiros, 16 psicólogos e 10 médicos, todos profissionais atuantes na cidade de Ribeirão Preto, com idades variando entre 20 e 48 anos. Todos eram ingênuos quanto aos propósitos do experimento.

Material. Foram montados dois blocos de papel contendo na primeira página instruções específicas para cada tipo de método psicofísico e na página seguinte uma lista de 15 quadros clínicos resultantes do estudo de KAMIZAKI et al. (1999) e caneta.

Procedimento. Os métodos psicofísicos utilizados foram estimação de magnitudes e estimação de categorias. O procedimento utilizado no primeiro método foi idêntico àquele descrito no estudo de KAMIZAKI et al. (1999), exceto que cada participante estabeleceu apenas 15 estimativas, sendo uma para cada um dos 15 quadros clínicos (diagnósticos) selecionados do estudo de KAMIZAKI et al. (1999). Estímulo padrão e módulo foram designados previamente, tendo sido estabelecido o valor arbitrário de 100 para Úlcera peptídica.
No segundo método, a tarefa dos participantes foi assinalar um escore, que variava de 1 a 7 , a cada quadro clínico, em função do grau de severidade dos quadros clínicos. O participante foi instruído a assinalar ao quadro clínico de maior gravidade o escore máximo de 7 e ao de menor gravidade, o escore mínimo de 1 . Os outros escores intermediários de 2 a 6 , deveriam ser utilizados para indicar graus intermediários de severidade que os quadros clínicos possuem. Previamente não foram estabelecidos os quadros clínicos de severidade máxima e mínima. Os diferentes quadros clínicos foram apresentados em uma série de 15, que foram dispostos em uma página, numa ordem totalmente aleatória para todos os participantes. Cada participante estabeleceu 15 estimativas, sendo uma para cada quadro clínico.

As instruções dadas para os participantes, independente do método psicofísico utilizado, requeriam que os julgamentos fossem realizados em função do grau relativo da gravidade dos quadros clínicos, considerando a maioria dos pacientes, cujo problema central é este quadro clínico e as outras condições relativamente controladas. Os mesmos 46 participantes fizeram as duas tarefas, sendo que metade julgou primeiro pelo método de estimação de magnitudes e depois pelo método de estimação de categorias, e a outra metade julgou na ordem inversa. $\mathrm{O}$ experimento foi realizado ora em laboratório, ora em residências, ora em locais de trabalho do participante. $\mathrm{O}$ instrumento era entregue e posteriormente recolhido, após os participantes serem instruídos a respondê-lo individualmente e sem interrupção. Adicionado a isso, os participantes foram instruídos a colocar a letra $\mathrm{D}$ no espaço correspondente às estimativas, caso não conhecesse algum diagnóstico listado e este quadro clínico não foi considerado no cômputo das médias.

\section{RESULTADOS E DISCUSSÃO}

Os dados apresentados na Tabela 1 de 100 quadros clínicos elaborada por KAMIZAKI et al. (1999) e os dados apresentados na Tabela 2 mostram os 15 quadros clínicos selecionados neste experimento, com suas respectivas médias geométricas (EM) e ordenação das posições (OP) de gravidade julgadas considerandose todas as amostras juntas. As estimativas médias para os diferentes quadros clínicos realizado pelos diferentes grupos de participantes (A1 amostra composta por enfermeiros; A2, por psicólogos e A3, por médicos) e as respectivas ordenações de posições estão apresentadas nas Tabelas 3 e 4 , respectivamente para os métodos psicofísicos utilizados: Estimação de categorias (EC) e Estimação de magnitudes (EM). 
Tabela 1 - Média geométrica das estimativas de magnitudes das amostras brasileiras (EM1, EM2, EM3) e americanas (EMM, EML), média geométrica geral das amostras brasileiras (EMB) e média geral americana (EMA) e respectivas ordenações de posições (OP) das gravidades julgadas de cada quadro clínico

\begin{tabular}{|c|c|c|c|c|c|c|c|c|c|c|c|c|c|c|}
\hline $\mathbf{A} 2$ & A3 & & ERAI & & $\mathbf{A M}$ & & $\mathbf{A L}$ & & SERAI & & & & & \\
\hline Diagnóstico & EM1 & OP & EM2 & OP & EM3 & OP & EMB & OP & EMM & OP & EML & OP & EMA & OP \\
\hline Caspa & 16,48 & $2^{\circ}$ & 1,90 & $1^{\mathrm{o}}$ & 17,83 & $1^{\circ}$ & 12,36 & $1^{\mathrm{o}}$ & 2,80 & $1^{\mathrm{o}}$ & 2,00 & $1^{\mathrm{o}}$ & 2,37 & $1^{\mathrm{o}}$ \\
\hline Verruga & 16,52 & $3^{\circ}$ & 3,79 & $2^{\circ}$ & 29,41 & $2^{\circ}$ & 16,96 & $2^{\circ}$ & 4,00 & $3^{\circ}$ & 6,00 & $3^{\circ}$ & 4,90 & $3^{\circ}$ \\
\hline Terçol & 16,31 & $1^{\circ}$ & 8,08 & $6^{\circ}$ & 35,18 & $4^{\circ}$ & 20,48 & $3^{\circ}$ & 10,60 & $7^{\circ}$ & 10,60 & $7^{\circ}$ & 10,60 & $7^{\circ}$ \\
\hline Acne & 17,69 & $4^{\circ}$ & 7,28 & $4^{\circ}$ & 34,48 & $3^{\circ}$ & 20,59 & $4^{\circ}$ & 23,00 & $18^{\circ}$ & 24,00 & $18^{\circ}$ & 23,49 & $18^{\circ}$ \\
\hline Ferida Comum & 30,63 & $9^{\circ}$ & 7,63 & $5^{\circ}$ & 35,58 & $6^{\circ}$ & 26,54 & $5^{\circ}$ & 7,00 & $2^{\circ}$ & 3,40 & $2^{\circ}$ & 4,88 & $2^{\circ}$ \\
\hline Período Pré-Menstrual & 20,87 & $5^{\circ}$ & 26,15 & $16^{\circ}$ & 35,40 & $5^{\circ}$ & 27,04 & $6^{\circ}$ & 29,20 & $24^{\circ}$ & 31,00 & $25^{\circ}$ & 30,00 & $25^{\circ}$ \\
\hline Resfriado & 25,95 & $8^{\circ}$ & 10,60 & $8^{\circ}$ & 42,31 & $7^{\circ}$ & 28,00 & $7^{\circ}$ & 10,20 & $4^{\circ}$ & 10,00 & $4^{\circ}$ & 10,00 & $4^{\circ}$ \\
\hline Astigmatismo & 34,40 & $11^{\circ}$ & 6,06 & $3^{\circ}$ & 45,02 & $10^{\circ}$ & 29,79 & $8^{\circ}$ & 14,20 & $8^{\circ}$ & 12,80 & $10^{\circ}$ & 13,48 & $10^{\circ}$ \\
\hline Menopausa & 24,87 & $7^{\circ}$ & 20,76 & $14^{\circ}$ & 51,02 & $11^{\circ}$ & 32,88 & $9^{\circ}$ & 24,60 & $13^{\circ}$ & 18,60 & $15^{\circ}$ & 21,39 & $15^{\circ}$ \\
\hline Furúnculo & 38,60 & $12^{\circ}$ & 11,77 & $9^{\circ}$ & 42,53 & $8^{\circ}$ & 33,70 & $10^{\circ}$ & 17,80 & $12^{\circ}$ & 18,40 & $11^{\circ}$ & 18,10 & $11^{\circ}$ \\
\hline Verminose & 39,27 & $13^{\circ}$ & 16,26 & $11^{\circ}$ & 44,84 & $9^{\circ}$ & 36,43 & $11^{\circ}$ & 13,00 & $10^{\circ}$ & 13,20 & $9^{\circ}$ & 13,10 & $9^{\circ}$ \\
\hline Miopia & 51,40 & $21^{\circ}$ & 8,26 & $7^{\circ}$ & 66,82 & $16^{\circ}$ & 43,62 & $12^{\circ}$ & 13,20 & $9^{\circ}$ & 12,80 & $8^{\circ}$ & 13,00 & $8^{\circ}$ \\
\hline Amidalite & 43,27 & $16^{\circ}$ & 27,37 & $18^{\circ}$ & 51,91 & $12^{\circ}$ & 43,68 & $13^{\circ}$ & 17,40 & $14^{\circ}$ & 21,80 & $13^{\circ}$ & 19,48 & $13^{\circ}$ \\
\hline Reação Ansiosa & 32,73 & $10^{\circ}$ & 52,77 & $38^{\circ}$ & 56,63 & $13^{\circ}$ & 44,51 & $14^{\circ}$ & 70,00 & $46^{\circ}$ & 60,20 & $47^{\circ}$ & 64,92 & $47^{\circ}$ \\
\hline Sinusite & 43,65 & $17^{\circ}$ & 22,59 & $15^{\circ}$ & 60,55 & $14^{\circ}$ & 45,48 & $15^{\circ}$ & 29,20 & $31^{\circ}$ & 35,60 & $28^{\circ}$ & 32,24 & $28^{\circ}$ \\
\hline Ulceração pelo Frio & 40,78 & $15^{\circ}$ & 26,67 & $17^{\circ}$ & 67,34 & $17^{\circ}$ & 48,07 & $16^{\circ}$ & 47,60 & $32^{\circ}$ & 40,40 & $37^{\circ}$ & 43,85 & $37^{\circ}$ \\
\hline Desvio Sexual & 24,13 & $6^{\circ}$ & 47,85 & $30^{\circ}$ & 124,60 & $52^{\circ}$ & 49,73 & $17^{\circ}$ & 99,00 & $47^{\circ}$ & 66,20 & $54^{\circ}$ & 80,96 & $54^{\circ}$ \\
\hline Luxação & 40,27 & $14^{\circ}$ & 16,01 & $10^{\circ}$ & 98,61 & $41^{\circ}$ & 51,39 & $18^{\circ}$ & 92,20 & $67^{\circ}$ & 110,20 & $65^{\circ}$ & 100,80 & $65^{\circ}$ \\
\hline Carbúnculo & 45,12 & $18^{\circ}$ & 20,00 & $12^{\circ}$ & 66,60 & $15^{\circ}$ & 55,15 & $19^{\circ}$ & 22,60 & $16^{\circ}$ & 23,40 & $17^{\circ}$ & 23,00 & $17^{\circ}$ \\
\hline Cachumba & 57,92 & $24^{\circ}$ & 31,57 & $21^{\circ}$ & 68,67 & $18^{\circ}$ & 56,66 & $20^{\circ}$ & 22,60 & $21^{\circ}$ & 27,60 & $20^{\circ}$ & 24,98 & $19^{\circ}$ \\
\hline Catapora & 60,31 & $25^{\circ}$ & 37,28 & $24^{\circ}$ & 72,61 & $21^{\circ}$ & 60,27 & $21^{\circ}$ & 21,00 & $17^{\circ}$ & 23,80 & $16^{\circ}$ & 22,36 & $16^{\circ}$ \\
\hline Infecção de Ouvido Médio & 54,61 & $22^{\circ}$ & 41,20 & $27^{\circ}$ & 76,67 & $25^{\circ}$ & 60,64 & $22^{\circ}$ & 27,20 & $26^{\circ}$ & 31,20 & $23^{\circ}$ & 29,13 & $23^{\circ}$ \\
\hline Insolação & 50,79 & $20^{\circ}$ & 47,24 & $29^{\circ}$ & 81,64 & $28^{\circ}$ & 61,49 & $23^{\circ}$ & 10,80 & $5^{\circ}$ & 10,20 & $5^{\circ}$ & 10,50 & $5^{\circ}$ \\
\hline Hérnia & 66,40 & $32^{\circ}$ & 28,69 & $19^{\circ}$ & 78,30 & $27^{\circ}$ & 62,86 & $24^{\circ}$ & 32,60 & $35^{\circ}$ & 41,00 & $29^{\circ}$ & 36,56 & $29^{\circ}$ \\
\hline Enxaqueca & 56,21 & $23^{\circ}$ & 53,85 & $40^{\circ}$ & 77,62 & $26^{\circ}$ & 64,07 & $25^{\circ}$ & 48,00 & $39^{\circ}$ & 47,60 & $40^{\circ}$ & 47,80 & $40^{\circ}$ \\
\hline Daltonismo & 76,90 & $44^{\circ}$ & 30,12 & $20^{\circ}$ & 69,85 & $19^{\circ}$ & 64,08 & $26^{\circ}$ & 19,20 & $11^{\circ}$ & 18,20 & $12^{\circ}$ & 18,69 & $12^{\circ}$ \\
\hline Ferida do Útero & 65,37 & $29^{\circ}$ & 39,15 & $25^{\circ}$ & 84,47 & $30^{\circ}$ & 67,40 & $27^{\circ}$ & 28,00 & $26^{\circ}$ & 31,00 & $24^{\circ}$ & 29,46 & $24^{\circ}$ \\
\hline Coqueluche & 62,90 & $26^{\circ}$ & 63,35 & $44^{\circ}$ & 75,77 & $24^{\circ}$ & 68,16 & $28^{\circ}$ & - & & - & & - & \\
\hline Hemorragia & 66,08 & $30^{\circ}$ & 54,88 & $42^{\circ}$ & 83,66 & $29^{\circ}$ & 70,81 & $29^{\circ}$ & 26,00 & $19^{\circ}$ & 24,20 & $19^{\circ}$ & 25,08 & $20^{\circ}$ \\
\hline Bursite & 66,09 & $31^{\circ}$ & 49,59 & $32^{\circ}$ & 85,27 & $31^{\circ}$ & 71,41 & $30^{\circ}$ & 34,80 & $33^{\circ}$ & 40,40 & $33^{\circ}$ & 37,50 & $30^{\circ}$ \\
\hline Esquizofrenia & 64,78 & $28^{\circ}$ & 100,40 & $55^{\circ}$ & 73,34 & $23^{\circ}$ & 72,04 & $31^{\circ}$ & 43,60 & $29^{\circ}$ & 32,60 & $36^{\circ}$ & 37,70 & $31^{\circ}$ \\
\hline Anemia & 81,40 & $50^{\circ}$ & 50,52 & $33^{\circ}$ & 72,76 & $22^{\circ}$ & 72,28 & $32^{\circ}$ & 56,00 & $38^{\circ}$ & 47,40 & $38^{\circ}$ & 51,52 & $43^{\circ}$ \\
\hline Sarampo & 69,27 & $36^{\circ}$ & 52,09 & $37^{\circ}$ & 87,39 & $35^{\circ}$ & 73,38 & $33^{\circ}$ & 24,80 & $22^{\circ}$ & 28,20 & $22^{\circ}$ & 26,45 & $21^{\circ}$ \\
\hline Bócio & 67,83 & $33^{\circ}$ & 40,72 & $26^{\circ}$ & 94,83 & $39^{\circ}$ & 73,97 & $34^{\circ}$ & 50,40 & $41^{\circ}$ & 50,40 & $41^{\circ}$ & 50,40 & $41^{\circ}$ \\
\hline Olho infectado & 71,85 & $40^{\circ}$ & 50,52 & $34^{\circ}$ & 88,62 & $36^{\circ}$ & 74,27 & $35^{\circ}$ & 45,20 & $28^{\circ}$ & 32,40 & $28^{\circ}$ & 38,27 & $33^{\circ}$ \\
\hline Veia Varicosa & 71,99 & $42^{\circ}$ & 53,13 & $39^{\circ}$ & 85,44 & $33^{\circ}$ & 76,33 & $36^{\circ}$ & 29,40 & $20^{\circ}$ & 27,40 & $21^{\circ}$ & 28,38 & $22^{\circ}$ \\
\hline Absesso Dental & 69,08 & $35^{\circ}$ & 51,19 & $35^{\circ}$ & 98,93 & $42^{\circ}$ & 77,16 & $37^{\circ}$ & 18,60 & $15^{\circ}$ & 22,00 & $15^{\circ}$ & 20,23 & $14^{\circ}$ \\
\hline Psoríase & 69,30 & $37^{\circ}$ & 36,84 & $23^{\circ}$ & 96,20 & $40^{\circ}$ & 77,24 & $38^{\circ}$ & 41,00 & $40^{\circ}$ & 50,20 & $40^{\circ}$ & 45,37 & $38^{\circ}$ \\
\hline Monoclease & 68,76 & $34^{\circ}$ & 80,00 & $48^{\circ}$ & 85,29 & $32^{\circ}$ & 79,21 & $39^{\circ}$ & 28,40 & $26^{\circ}$ & 32,00 & $27^{\circ}$ & 30,15 & $26^{\circ}$ \\
\hline Gonorréia & 82,32 & $52^{\circ}$ & 97,03 & $53^{\circ}$ & 71,85 & $20^{\circ}$ & 79,62 & $40^{\circ}$ & 36,00 & $27^{\circ}$ & 40,80 & $34^{\circ}$ & 38,32 & $34^{\circ}$ \\
\hline Pressão Baixa & 79,03 & $46^{\circ}$ & 64,92 & $45^{\circ}$ & 86,88 & $34^{\circ}$ & 79,76 & $41^{\circ}$ & 19,60 & $44^{\circ}$ & 56,20 & $44^{\circ}$ & 37,80 & $32^{\circ}$ \\
\hline Frigidez & 46,98 & $19^{\circ}$ & 77,10 & $46^{\circ}$ & 134,27 & $57^{\circ}$ & 79,97 & $42^{\circ}$ & 71,60 & $54^{\circ}$ & 82,00 & $54^{\circ}$ & 76,62 & $51^{\circ}$ \\
\hline Cisto Ovariano & 71,89 & $41^{\circ}$ & 48,80 & $31^{\circ}$ & 107,85 & $46^{\circ}$ & 81,53 & $43^{\circ}$ & 47,60 & $37^{\circ}$ & 46,00 & $39^{\circ}$ & 46,79 & $39^{\circ}$ \\
\hline
\end{tabular}




\begin{tabular}{|c|c|c|c|c|c|c|c|c|c|c|c|c|c|c|}
\hline $\mathbf{A 2}$ & A3 & & GERAI & & $\mathbf{A M}$ & & $\mathbf{A L}$ & & GERAI & & & & & \\
\hline Diagnóstico & EM1 & OP & EM2 & OP & EM3 & OP & EMB & OP & EMM & OP & EML & OP & EMA & OP \\
\hline Artrite & 63,94 & $27^{\circ}$ & 45,94 & $28^{\circ}$ & 130,14 & $55^{\circ}$ & 82,36 & $44^{\circ}$ & 101,40 & $63^{\circ}$ & 94,20 & $63^{\circ}$ & 97,73 & $63^{\circ}$ \\
\hline Gota & 69,67 & $38^{\circ}$ & 51,72 & $36^{\circ}$ & 122,86 & $51^{\circ}$ & 84,84 & $45^{\circ}$ & 50,40 & $42^{\circ}$ & 50,40 & $42^{\circ}$ & 50,40 & $42^{\circ}$ \\
\hline Varíola & 73,05 & $43^{\circ}$ & 55,64 & $43^{\circ}$ & 119,39 & $50^{\circ}$ & 85,23 & $46^{\circ}$ & 133,00 & $74^{\circ}$ & 131,40 & $74^{\circ}$ & 132,20 & $73^{\circ}$ \\
\hline Herpes & 78,51 & $45^{\circ}$ & 79,53 & $47^{\circ}$ & 106,61 & $45^{\circ}$ & 90,10 & $47^{\circ}$ & 41,60 & $30^{\circ}$ & 35,40 & $30^{\circ}$ & 38,37 & $35^{\circ}$ \\
\hline Surdez & 80,01 & $48^{\circ}$ & 36,31 & $22^{\circ}$ & 148,14 & $62^{\circ}$ & 92,45 & $48^{\circ}$ & 105,20 & $70^{\circ}$ & 121,40 & $70^{\circ}$ & 113,01 & $69^{\circ}$ \\
\hline Asma & 80,48 & $49^{\circ}$ & 54,56 & $41^{\circ}$ & 130,12 & $54^{\circ}$ & 93,48 & $49^{\circ}$ & 91,40 & $57^{\circ}$ & 89,00 & $57^{\circ}$ & 90,19 & $59^{\circ}$ \\
\hline Bronquite & 91,60 & $57^{\circ}$ & 89,72 & $51^{\circ}$ & 100,51 & $44^{\circ}$ & 94,88 & $50^{\circ}$ & 37,20 & $36^{\circ}$ & 44,20 & $36^{\circ}$ & 40,55 & $36^{\circ}$ \\
\hline Queimadura & 95,88 & $60^{\circ}$ & 117,81 & $61^{\circ}$ & 94,28 & $38^{\circ}$ & 98,30 & $51^{\circ}$ & 82,80 & $52^{\circ}$ & 76,00 & $52^{\circ}$ & 79,33 & $52^{\circ}$ \\
\hline Pedra na Vesícula & 87,25 & $54^{\circ}$ & 102,64 & $58^{\circ}$ & 110,73 & $47^{\circ}$ & 99,20 & $52^{\circ}$ & 82,80 & $59^{\circ}$ & 90,40 & $59^{\circ}$ & 86,52 & $57^{\circ}$ \\
\hline Febre do Feno & 102,52 & $62^{\circ}$ & 223,61 & $87^{\circ}$ & 89,12 & $37^{\circ}$ & 99,92 & $53^{\circ}$ & 32,80 & $23^{\circ}$ & 28,40 & $23^{\circ}$ & 30,52 & $27^{\circ}$ \\
\hline Úlcera peptidica & 100,00 & $61^{\circ}$ & 100,00 & $54^{\circ}$ & 100,00 & $43^{\circ}$ & 100,00 & $54^{\circ}$ & 100,00 & $65^{\circ}$ & 100,00 & $65^{\circ}$ & 100,00 & $64^{\circ}$ \\
\hline Obesidade & \begin{tabular}{|l|}
79,84 \\
\end{tabular} & $47^{\circ}$ & 96,86 & $52^{\circ}$ & 126,51 & $53^{\circ}$ & 100,44 & $55^{\circ}$ & 70,20 & $48^{\circ}$ & 69,00 & $48^{\circ}$ & 69,60 & $49^{\circ}$ \\
\hline Pneumonia & 91,30 & $56^{\circ}$ & 119,38 & $62^{\circ}$ & 113,67 & $48^{\circ}$ & 104,31 & $56^{\circ}$ & 59,60 & $45^{\circ}$ & 57,20 & $45^{\circ}$ & 58,39 & $45^{\circ}$ \\
\hline Hipertensão & 81,78 & $51^{\circ}$ & 108,37 & $59^{\circ}$ & 133,24 & $56^{\circ}$ & 104,92 & $57^{\circ}$ & 91,20 & $61^{\circ}$ & 92,00 & $61^{\circ}$ & 91,60 & $60^{\circ}$ \\
\hline Apendicite & 85,01 & $53^{\circ}$ & 84,20 & $49^{\circ}$ & 149,50 & $63^{\circ}$ & 108,50 & $58^{\circ}$ & 59,60 & $53^{\circ}$ & 78,20 & $53^{\circ}$ & 68,27 & $48^{\circ}$ \\
\hline Glaucoma & 70,62 & $39^{\circ}$ & 101,11 & $56^{\circ}$ & 173,30 & $70^{\circ}$ & 109,16 & $59^{\circ}$ & 79,60 & $58^{\circ}$ & 89,20 & $58^{\circ}$ & 84,26 & $56^{\circ}$ \\
\hline Esgotamento Nervoso & 107,95 & $66^{\circ}$ & 109,77 & $60^{\circ}$ & 116,32 & $49^{\circ}$ & 111,80 & $60^{\circ}$ & 120,20 & $64^{\circ}$ & 99,00 & $64^{\circ}$ & 109,09 & $66^{\circ}$ \\
\hline Depressão & 92,59 & $58^{\circ}$ & 136,08 & $65^{\circ}$ & 137,41 & $58^{\circ}$ & 115,99 & $61^{\circ}$ & 88,20 & $51^{\circ}$ & 73,40 & $51^{\circ}$ & 80,46 & $53^{\circ}$ \\
\hline Impotência Sexual & 107,03 & $65^{\circ}$ & 101,11 & $57^{\circ}$ & 143,10 & $59^{\circ}$ & 120,08 & $62^{\circ}$ & 79,60 & $56^{\circ}$ & 84,60 & $56^{\circ}$ & 82,06 & $55^{\circ}$ \\
\hline Pressão Alta & 93,04 & $59^{\circ}$ & 126,07 & $63^{\circ}$ & 167,70 & $68^{\circ}$ & 125,89 & $63^{\circ}$ & 114,20 & $69^{\circ}$ & 120,60 & $69^{\circ}$ & 117,36 & $70^{\circ}$ \\
\hline Sífilis & 105,97 & $64^{\circ}$ & 151,12 & $70^{\circ}$ & 145,84 & $61^{\circ}$ & 128,51 & $64^{\circ}$ & 74,40 & $50^{\circ}$ & 70,40 & $50^{\circ}$ & 72,37 & $50^{\circ}$ \\
\hline Uremia & 87,76 & $55^{\circ}$ & 20,00 & $13^{\circ}$ & 210,96 & $82^{\circ}$ & 131,12 & $65^{\circ}$ & 218,00 & $98^{\circ}$ & 218,00 & $98^{\circ}$ & 218,00 & $98^{\circ}$ \\
\hline Queimadura do Sol & 120,81 & $72^{\circ}$ & 87,22 & $50^{\circ}$ & 164,99 & $67^{\circ}$ & 131,49 & $66^{\circ}$ & 10,80 & $6^{\circ}$ & 10,20 & $6^{\circ}$ & 10,50 & $6^{\circ}$ \\
\hline Envenenamento Acidental & 112,27 & $68^{\circ}$ & 156,02 & $71^{\circ}$ & 157,01 & $65^{\circ}$ & 136,00 & $67^{\circ}$ & 91,80 & $55^{\circ}$ & 82,00 & $55^{\circ}$ & 86,76 & $58^{\circ}$ \\
\hline Mal de Parkinson & 111,93 & $67^{\circ}$ & 170,21 & $76^{\circ}$ & 169,19 & $69^{\circ}$ & 140,92 & $68^{\circ}$ & 141,00 & $81^{\circ}$ & 146,80 & $80^{\circ}$ & 143,87 & $76^{\circ}$ \\
\hline Distrofia Muscular & 103,24 & $63^{\circ}$ & 157,04 & $72^{\circ}$ & 206,69 & $78^{\circ}$ & 146,36 & $69^{\circ}$ & 164,00 & $93^{\circ}$ & 167,00 & $93^{\circ}$ & 165,49 & $91^{\circ}$ \\
\hline Tuberculose & 132,93 & $78^{\circ}$ & 139,35 & $66^{\circ}$ & 177,02 & $72^{\circ}$ & 150,71 & $70^{\circ}$ & 122,60 & $71^{\circ}$ & 121,40 & $71^{\circ}$ & 122,00 & $71^{\circ}$ \\
\hline Insuficiência Renal & 155,13 & $87^{\circ}$ & 168,02 & $75^{\circ}$ & 143,62 & $60^{\circ}$ & 151,85 & $71^{\circ}$ & 59,60 & $49^{\circ}$ & 70,20 & $49^{\circ}$ & 64,68 & $46^{\circ}$ \\
\hline Diabetis & 119,14 & $71^{\circ}$ & 142,62 & $68^{\circ}$ & 199,89 & $77^{\circ}$ & 152,52 & $72^{\circ}$ & 135,00 & $73^{\circ}$ & 130,40 & $73^{\circ}$ & 132,68 & $74^{\circ}$ \\
\hline Epilepsia & 118,19 & $70^{\circ}$ & 142,87 & $69^{\circ}$ & 208,83 & $80^{\circ}$ & 154,90 & $73^{\circ}$ & 112,80 & $68^{\circ}$ & 112,40 & $68^{\circ}$ & 112,60 & $68^{\circ}$ \\
\hline Coágulo Vasos Sangüíneos & 147,32 & $84^{\circ}$ & 188,60 & $80^{\circ}$ & 155,12 & $64^{\circ}$ & 155,78 & $74^{\circ}$ & 164,00 & $77^{\circ}$ & 142,60 & $77^{\circ}$ & 152,93 & $82^{\circ}$ \\
\hline Endurecimento das Artérias & 128,52 & $75^{\circ}$ & 187,54 & $79^{\circ}$ & 177,21 & $73^{\circ}$ & 155,88 & $75^{\circ}$ & 120,40 & $66^{\circ}$ & 103,80 & $66^{\circ}$ & 111,79 & $67^{\circ}$ \\
\hline Colapso Pulmonar & 146,68 & $83^{\circ}$ & 195,57 & $82^{\circ}$ & 158,24 & $66^{\circ}$ & 158,12 & $76^{\circ}$ & 90,00 & $62^{\circ}$ & 93,60 & $62^{\circ}$ & 91,78 & $61^{\circ}$ \\
\hline Adição de Drogas & 144,49 & $82^{\circ}$ & 158,56 & $73^{\circ}$ & 177,81 & $74^{\circ}$ & 160,38 & $77^{\circ}$ & 147,00 & $79^{\circ}$ & 144,80 & $79^{\circ}$ & 145,90 & $77^{\circ}$ \\
\hline Hepatite & 141,79 & $81^{\circ}$ & 140,16 & $67^{\circ}$ & 191,79 & $76^{\circ}$ & 160,96 & $78^{\circ}$ & 97,00 & $60^{\circ}$ & 90,40 & $60^{\circ}$ & 93,64 & $62^{\circ}$ \\
\hline Enfisema & 123,07 & $73^{\circ}$ & 163,21 & $74^{\circ}$ & 208,41 & $79^{\circ}$ & 161,50 & $79^{\circ}$ & 137,40 & $75^{\circ}$ & 136,40 & $75^{\circ}$ & 136,90 & $75^{\circ}$ \\
\hline Psicose Maníaco-Depressiva & 131,45 & $76^{\circ}$ & 249,80 & $91^{\circ}$ & 176,03 & $71^{\circ}$ & 163,05 & $80^{\circ}$ & 160,00 & $89^{\circ}$ & 161,80 & $89^{\circ}$ & 160,90 & $90^{\circ}$ \\
\hline Mordida de Cobra & 132,67 & $77^{\circ}$ & 129,40 & $64^{\circ}$ & 222,70 & $84^{\circ}$ & 163,70 & $81^{\circ}$ & 64,40 & $43^{\circ}$ & 52,00 & $44^{\circ}$ & 57,87 & $44^{\circ}$ \\
\hline Esquizofrenia & 128,29 & $74^{\circ}$ & 177,84 & $77^{\circ}$ & 219,91 & $83^{\circ}$ & 169,40 & $82^{\circ}$ & 170,40 & $92^{\circ}$ & 166,80 & $92^{\circ}$ & 168,59 & $93^{\circ}$ \\
\hline Cegueira & 114,34 & $69^{\circ}$ & 211,93 & $84^{\circ}$ & 227,85 & $86^{\circ}$ & 169,51 & $83^{\circ}$ & 148,40 & $90^{\circ}$ & 162,20 & $90^{\circ}$ & 155,15 & $85^{\circ}$ \\
\hline Pancreatite & 136,91 & $80^{\circ}$ & 187,23 & $78^{\circ}$ & 210,73 & $81^{\circ}$ & 171,58 & $84^{\circ}$ & 124,80 & $72^{\circ}$ & 121,40 & $72^{\circ}$ & 123,09 & $72^{\circ}$ \\
\hline Coágulo nos Pulmões & 183,09 & $91^{\circ}$ & 239,35 & $88^{\circ}$ & 178,17 & $75^{\circ}$ & 188,57 & $85^{\circ}$ & 164,00 & $78^{\circ}$ & 142,60 & $78^{\circ}$ & 152,93 & $83^{\circ}$ \\
\hline Problema Card.Congênito & 156,19 & $89^{\circ}$ & 195,20 & $81^{\circ}$ & 233,23 & $87^{\circ}$ & 191,50 & $86^{\circ}$ & 167,00 & $82^{\circ}$ & 150,20 & $82^{\circ}$ & 158,38 & $88^{\circ}$ \\
\hline Retardo Mental & 148,65 & $85^{\circ}$ & 239,35 & $89^{\circ}$ & 227,67 & $85^{\circ}$ & 192,37 & $87^{\circ}$ & 150,40 & $91^{\circ}$ & 164,20 & $91^{\circ}$ & 157,15 & $87^{\circ}$ \\
\hline Esclerose & 136,30 & $79^{\circ}$ & 220,93 & $86^{\circ}$ & 263,35 & $89^{\circ}$ & 194,80 & $88^{\circ}$ & 182,00 & $94^{\circ}$ & 172,60 & $94^{\circ}$ & 177,24 & $94^{\circ}$ \\
\hline Derrame & 155,99 & $88^{\circ}$ & 211,21 & $83^{\circ}$ & 244,01 & $88^{\circ}$ & 198,43 & $89^{\circ}$ & 153,20 & $85^{\circ}$ & 151,80 & $85^{\circ}$ & 152,50 & $81^{\circ}$ \\
\hline Cirrose Hepática & 154,27 & $86^{\circ}$ & 218,79 & $85^{\circ}$ & 270,74 & $91^{\circ}$ & 206,46 & $90^{\circ}$ & 145,00 & $81^{\circ}$ & 148,80 & $81^{\circ}$ & 146,89 & $78^{\circ}$ \\
\hline Infecção Cerebral & 184,67 & $92^{\circ}$ & 300,00 & $93^{\circ}$ & 270,36 & $90^{\circ}$ & 233,38 & $91^{\circ}$ & 177,00 & $88^{\circ}$ & 159,60 & $88^{\circ}$ & 168,07 & $92^{\circ}$ \\
\hline Ataque Cardíaco & 178,47 & $90^{\circ}$ & 309,17 & $94^{\circ}$ & 282,75 & $92^{\circ}$ & 237,01 & $92^{\circ}$ & 170,00 & $86^{\circ}$ & 151,80 & $86^{\circ}$ & 160,64 & $89^{\circ}$ \\
\hline Tumor & 193,09 & $94^{\circ}$ & 239,35 & $90^{\circ}$ & 299,75 & $93^{\circ}$ & 241,54 & $93^{\circ}$ & 162,20 & $83^{\circ}$ & 151,60 & $83^{\circ}$ & 156,81 & $88^{\circ}$ \\
\hline Paralisia Cerebral & 185,05 & $93^{\circ}$ & 322,14 & $95^{\circ}$ & 322,39 & $95^{\circ}$ & 256,30 & $94^{\circ}$ & 151,80 & $87^{\circ}$ & 156,60 & $87^{\circ}$ & 154,18 & $84^{\circ}$ \\
\hline Falência Cardíaca & 196,63 & $95^{\circ}$ & 276,82 & $92^{\circ}$ & 337,04 & $96^{\circ}$ & 258,39 & $95^{\circ}$ & 154,80 & $76^{\circ}$ & 141,20 & $76^{\circ}$ & 147,84 & $79^{\circ}$ \\
\hline Hemorragia Cerebral & 204,72 & $97^{\circ}$ & 336,25 & $97^{\circ}$ & 300,31 & $94^{\circ}$ & 258,64 & $96^{\circ}$ & 176,00 & $93^{\circ}$ & 206.00 & $96^{\circ}$ & 190,41 & $95^{\circ}$ \\
\hline Coma & 203,33 & $96^{\circ}$ & 324,02 & $96^{\circ}$ & 385,06 & $100^{\circ}$ & 284,15 & $97^{\circ}$ & 152,60 & $84^{\circ}$ & 151,60 & $84^{\circ}$ & 152,10 & $80^{\circ}$ \\
\hline Leucemia & 207,60 & $100^{\circ}$ & 363,82 & $98^{\circ}$ & 371,28 & $98^{\circ}$ & 289,04 & $98^{\circ}$ & 232,00 & $95^{\circ}$ & 177,40 & $95^{\circ}$ & 202,87 & $96^{\circ}$ \\
\hline Cancer & 206,51 & $98^{\circ}$ & 389,09 & $99^{\circ}$ & 373,47 & $99^{\circ}$ & 292,02 & $99^{\circ}$ & 210,00 & 97 & 216,20 & $97^{\circ}$ & 213,08 & $97^{\circ}$ \\
\hline AIDS & 206,89 & $99^{\circ}$ & 572,51 & $100^{\circ}$ & 337,77 & $97^{\circ}$ & 295,76 & $100^{\circ}$ & - & & - & & - & \\
\hline
\end{tabular}

Obs: Para propósito de comparação as médias geométricas das amostras AM e AL foram divididas por 5, pois no estudo WYLER et al. (1968) foi adotado o módulo 500 para Úlcera peptídica, enquanto que em nosso estudo foi 100 
Tabela 2 - Média geométrica das estimativas de magnitudes (EM) e ordenação das posições (OP) de gravidade julgadas de cada quadro clínico (diagnóstico) considerando todas as amostras juntas do Estudo de KAMIZAKI et al. (1999)

\begin{tabular}{l|c|c}
\hline \multicolumn{1}{c|}{ Diagnósticos } & E M & OP \\
\hline Caspa & 12,53 & $1^{\circ}$ \\
\hline Furúnculo & 33,70 & $10^{\circ}$ \\
\hline Período pré-menstrual & 27,04 & $6^{\circ}$ \\
\hline Insolaçăo & 61,49 & $23^{\circ}$ \\
\hline Sarampo & 73,38 & $33^{\circ}$ \\
\hline Amidalite & 43,68 & $13^{\circ}$ \\
\hline Equizema & 72,04 & $31^{\circ}$ \\
\hline Úlcera Peptidica & 100,00 & $54^{\circ}$ \\
\hline Esgotamento nervoso & 111,80 & $60^{\circ}$ \\
\hline Hipertireoidismo & 104,92 & $68^{\circ}$ \\
\hline Epilepsia & 154,90 & $73^{\circ}$ \\
\hline Colapso pulmonar & 158,12 & $76^{\circ}$ \\
\hline Psicose maníaco depressiva & 163,05 & $80^{\circ}$ \\
\hline Falência cardíaca & 258,39 & $95^{\circ}$ \\
\hline Coma & 284,15 & $97^{\circ}$ \\
\hline
\end{tabular}

Tabela 3 - Média aritmética das estimativas de categorias (EC) e ordenação das posições (OP) de gravidade julgadas de cada quadro clínico (diagnóstico) das diferentes amostras (A1, A2 e A3)

\begin{tabular}{l|c|c|c|c|c|c}
\multicolumn{1}{c}{ Al } & \multicolumn{3}{c}{ A2 } & \multicolumn{3}{c}{ A3 } \\
\hline \multicolumn{1}{c}{ Diagnósticos } & EC & OP & EC & OP & EC & OP \\
\hline Ulcera Peptídica & 4,50 & $10^{\circ}$ & 4,12 & $9^{\circ}$ & 4,00 & $10^{\circ}$ \\
\hline Epilepsia & 4,80 & $11^{\circ}$ & 5,18 & $11^{\circ}$ & 5,10 & $11^{\circ}$ \\
\hline Cæspa & 1,30 & $1^{\circ}$ & 1,18 & $1^{\circ}$ & 1,10 & $1^{\circ}$ \\
\hline Falência cardiaca & 6,75 & $15^{\circ}$ & 6,18 & $14^{\circ}$ & 6,50 & $15^{\circ}$ \\
\hline Arridalite & 2,95 & $5^{\circ}$ & 2,82 & $6^{\circ}$ & 1,90 & $6^{\circ}$ \\
\hline Colapso pulmonar & 6,55 & $14^{\circ}$ & 6,00 & $13^{\circ}$ & 6,00 & $13^{\circ}$ \\
\hline Furínculo & 2,10 & $2^{\circ}$ & 1,53 & $2^{\circ}$ & 1,80 & $3^{\circ}$ \\
\hline Sarampo & 4,15 & $7^{\circ}$ & 2,53 & $5^{\circ}$ & 2,20 & $8^{\circ}$ \\
\hline Hipertensão & 4,15 & $8^{\circ}$ & 3,59 & $8^{\circ}$ & 3,90 & $9^{\circ}$ \\
\hline $\begin{array}{l}\text { Psicose Maríaco } \\
\text { Depressiva }\end{array}$ & 5,20 & $12^{\circ}$ & 5,94 & $12^{\circ}$ & 5,10 & $12^{\circ}$ \\
\hline Insolação & 3,35 & $6^{\circ}$ & 2,12 & $4^{\circ}$ & 1,80 & $4^{\circ}$ \\
\hline Equizema & 2,55 & $4^{\circ}$ & 3,15 & $7^{\circ}$ & 1,80 & $5^{\circ}$ \\
\hline Coma & 6,50 & $13^{\circ}$ & 6,35 & $15^{\circ}$ & 6,30 & $14^{\circ}$ \\
\hline Esgotamento & 4,20 & $9^{\circ}$ & 4,12 & $10^{\circ}$ & 2,00 & $7^{\circ}$ \\
Nervoso & & & & & & \\
\hline $\begin{array}{l}\text { Período Pré- } \\
\text { Menstrual }\end{array}$ & 2,15 & $3^{\circ}$ & 2,00 & $3^{\circ}$ & 1,30 & $2^{\circ}$ \\
\hline & & & & & & \\
\hline
\end{tabular}

Tabela 4 - Média geométrica das estimativas de magnitudes (EM) e ordenação das posições (OP) de gravidade julgadas de cada quadro clínico (diagnóstico) das diferentes amostras (A1, A2 e A3)

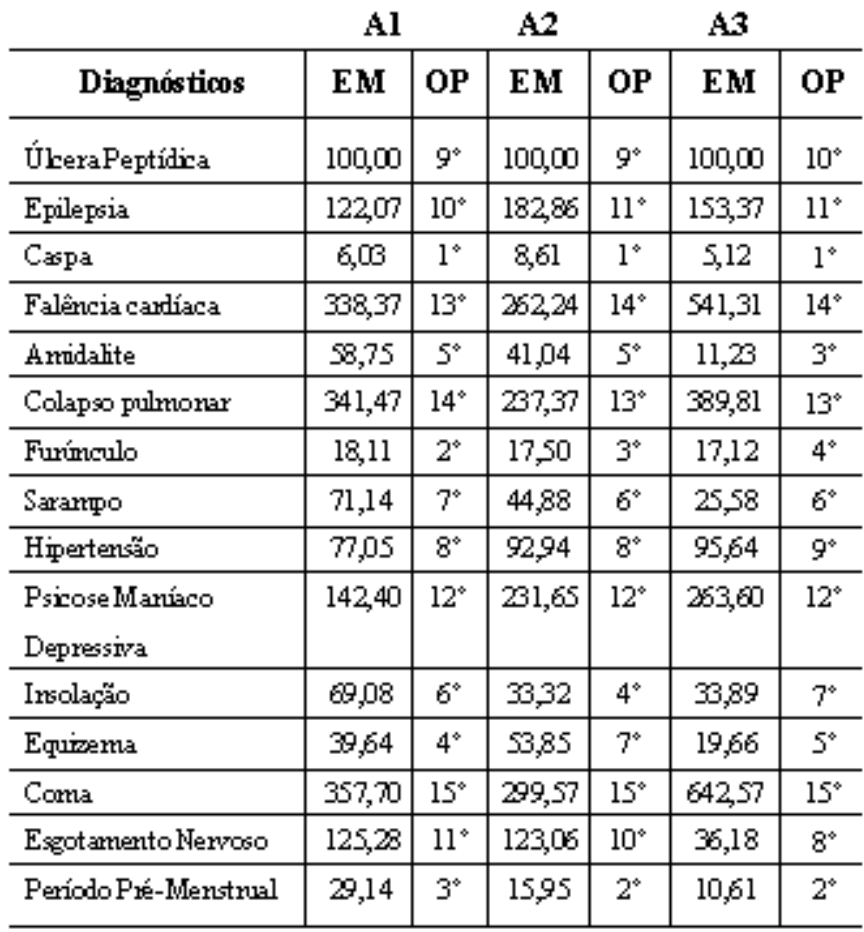

Tabela 5 - Média geométrica das estimativas de magnitudes (EM), média aritmética das estimativas de categorias (EC) e ordenação das posições (OP) de gravidade julgada de cada quadro clínico considerando todas as amostras juntas

\begin{tabular}{l|c|c|c|c}
\hline \multicolumn{1}{c|}{ Diagnósticos } & EM & OP & EC & OP \\
\hline Úlcera peptídica & 100,00 & $10^{\circ}$ & 4,26 & $10^{\circ}$ \\
\hline Epilepsia & 148,31 & $11^{\circ}$ & 5,00 & $11^{\circ}$ \\
\hline Cspa & 6,62 & $1^{\circ}$ & 1,21 & $1^{\circ}$ \\
\hline Falência cardíaca & 341,01 & $14^{\circ}$ & 6,49 & $15^{\circ}$ \\
\hline Amidalite & 36,29 & $4^{\circ}$ & 2,68 & $6^{\circ}$ \\
\hline Colapso pulmonar & 307,94 & $13^{\circ}$ & 6,23 & $13^{\circ}$ \\
\hline Furínculo & 17,67 & $2^{\circ}$ & 1,83 & $2^{\circ}$ \\
\hline Sarampo & 48,44 & $7^{\circ}$ & 3,15 & $7^{\circ}$ \\
\hline Hipertensão & 86,34 & $8^{\circ}$ & 3,89 & $9^{\circ}$ \\
\hline Psicose maríaco depressiva & 193,57 & $12^{\circ}$ & 5,45 & $12^{\circ}$ \\
\hline Insolação & 45,60 & $6^{\circ}$ & 2,57 & $5^{\circ}$ \\
\hline Equizema & 36,94 & $5^{\circ}$ & 2,56 & $4^{\circ}$ \\
\hline Coma & 380,00 & $15^{\circ}$ & 6,40 & $14^{\circ}$ \\
\hline Esgotamento nervoso & 95,56 & $9^{\circ}$ & 3,70 & $8^{\circ}$ \\
\hline Período pré-menstrual & 18,90 & $3^{\circ}$ & 1,91 & $3^{\circ}$ \\
\hline
\end{tabular}

Pode-se observar nestas Tabelas, que independente do método psicofísico utilizado, os quadros clínicos caspa, furúnculo e período pré-menstrual foram considerados os de menor gravidade enquanto que, os quadros clínicos coma, falência cardíaca e colapso 
pulmonar, os de maior gravidade. De fato, o coeficiente de concordância de Kendall (W) mostrou que, as diferentes amostras foram altamente concordantes quanto à ordenação desses diferentes quadros clínicos. Esses valores do coeficiente de concordância foram $\mathrm{W}=0,96$ para todos os quadros clínicos, 0,92 para o método de estimação de categorias, 0,97 para o de estimação de magnitudes, sendo ambos altamente significativos $(\mathrm{p}<0,001)$.

Como conseqüência dessa alta concordância dos julgamentos feitos pelas diferentes amostras, as estimativas de todos os participantes foram agrupadas em separado, para cada método (essas estimativas juntamente com suas respectivas ordenações estão apresentadas na Tabela 5). Pode-se observar nitidamente que estas ordenações, considerando-se cada método, não foram substancialmente diferentes daquelas produzidas pelas diferentes amostras, conforme apresentadas nas Tabelas 3 e 4. Como antes coma, falência cardíaca, colapso pulmonar, psicose maníaco depressiva e epilepsia foram considerados os quadros de maior gravidade, enquanto que, os quadros clínicos caspa, furúnculo, período pré-menstrual, equizema e amidalite foram considerados os de menor gravidade.

O cálculo do coeficiente de concordância tomando-se os dados de estimativas efetuadas por meio de cada método, para os 15 quadros clínicos, mostrou $\mathrm{W}=0,99(\mathrm{p}<0,001)$, indicando, portanto, que a ordenação por postos derivada das estimativas de cada método são altamente concordantes. Por exemplo, o quadro clínico período pré-menstrual ocupa a $3^{\mathrm{a}}$ posição e o quadro clínico colapso pulmonar ocupa a $13^{\mathrm{a}}$ posição nos dois tipos de contínuos de respostas. A análise que se segue foi feita considerando-se as estimativas médias apresentadas na Tabela 5. Para verificar se o contínuo de gravidade de quadros clínicos tem características protéticas ou metatéticas, como ocorre com os contínuos sensoriais, as médias aritméticas das estimativas de categorias foram projetadas em função das médias geométricas das estimativas de magnitudes dos quadros clínicos. Em coordenadas lineares a relação entre essas estimativas mostrou uma leve concavidade descendente. Também, quando as médias aritméticas das estimativas de categorias foram projetadas em função dos logaritmos das médias geométricas das estimativas de magnitudes, a relação mostrou uma concavidade ascendente. A Figura 1 mostra claramente esta relação em coordenadas lineares e a Figura 2 em coordenadas mono-logarítmicas. Tomadas juntas, fica demonstrado que o contínuo de gravidade de quadros clínicos tem características de contínuo protético ou aditivo.

Outro resultado interessante é a relação entre os desvios-padrão da média geométrica e as médias geométricas das estimativas de magnitudes (ver Figura
3). Observa-se que a relação é linear, de modo que quanto maior a estimativa de magnitude, tanto maior é o desvio padrão da média. Portanto, este padrão de resultados mostra o princípio geral conhecido como Lei de Ekman (STEVENS, 1966, 1975) de que a variabilidade das estimativas é uma função linear da magnitude das estimativas. De outro lado, a mesma relação não ocorre com as estimativas de categorias, uma vez que nas categorias extremas, geralmente o desvio padrão aritmético é pequeno, e no restante, usualmente é constante ou aumenta em função da categoria média (ver Figura 4). Para detalhes referentes às Figuras projetadas em função dos dados das Amostras 1, 2 e 3.

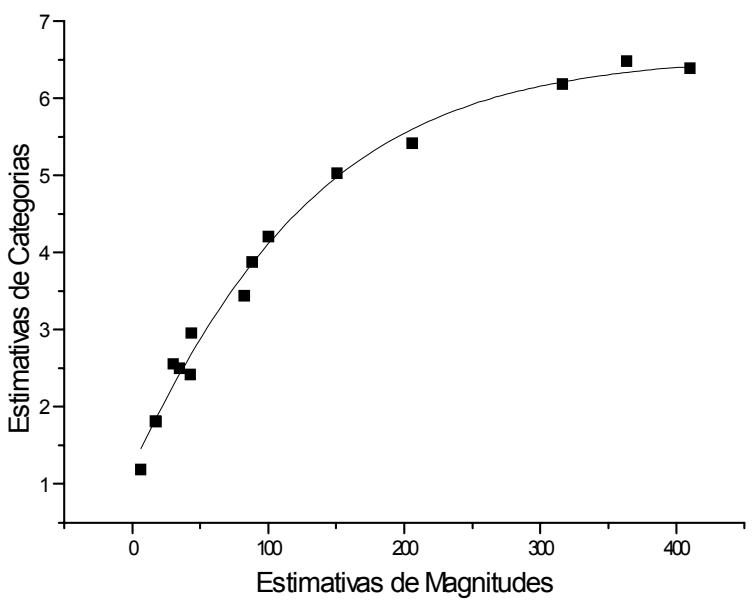

Figura 1 - Relação entre as médias aritméticas das estimativas de categorias e as médias geométricas das estimativas de magnitudes da gravidade de quadros clínicos em coordenadas lineares

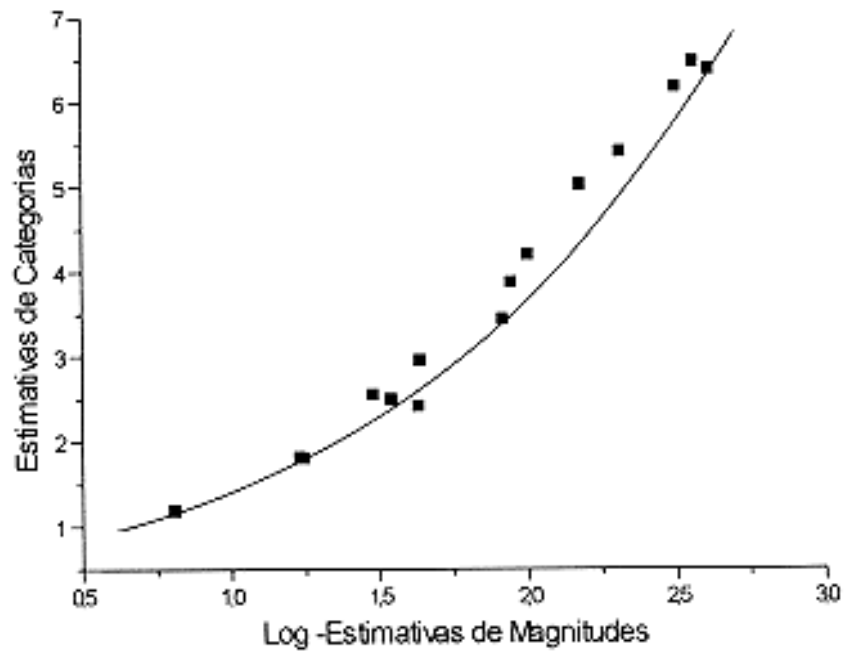

Figura 2 - Relação entre as médias aritméticas das estimativas de categorias e os logarítmos das médias geométricas das estimativas de magnitudes da gravidade de quadros clínicos 


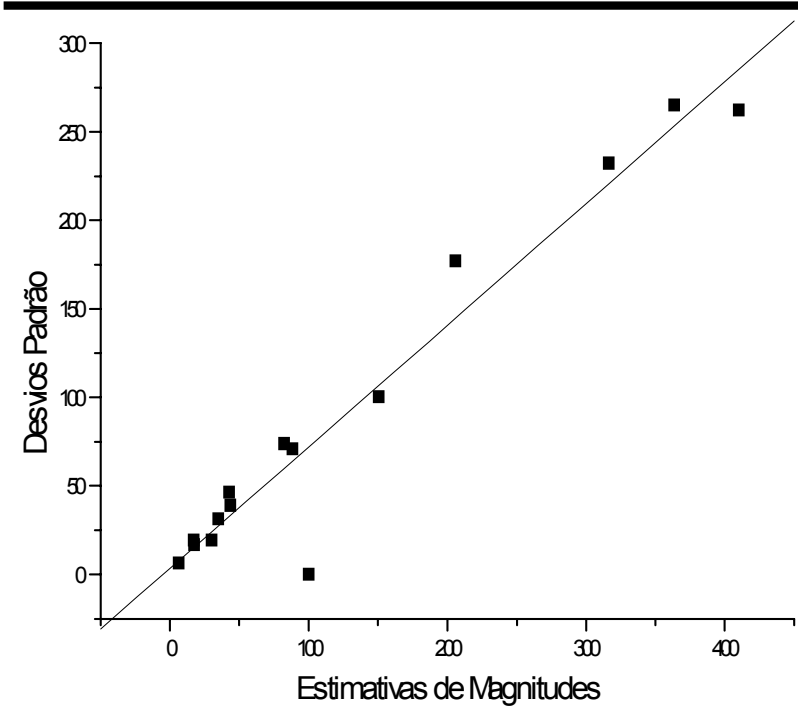

Figura 3 - Desvio padrão da média geométrica em função da média geométrica das estimativas de magnitudes da gravidade de quadros clínicos

Todavia, existem algumas diferenças fundamentais nos escalonamentos obtidos. Com o método de estimação de categorias podemos apenas obter a ordenação e a diferença entre os graus de gravidade. Baseado nesse método, não podemos afirmar o quanto o grau de gravidade de um dado quadro clínico é maior ou menor em comparação ao grau de um outro. Com o método de estimação de magnitudes, podemos obter a ordenação, a diferença e, também, as razões entre os graus

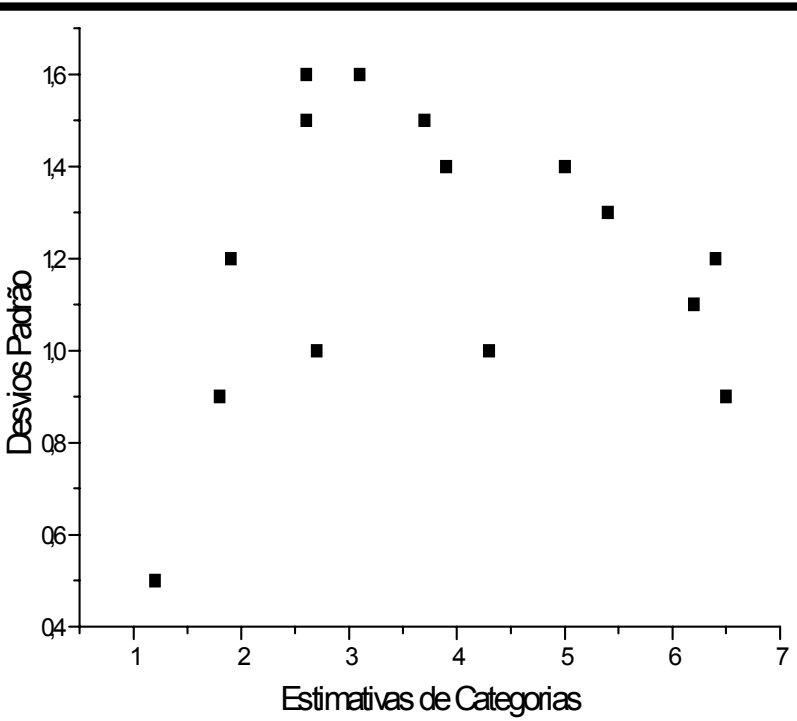

Figura 4 - Desvio padrão da média aritmética em função da média aritmética das estimativas de categorias da gravidade de quadros clínicos

de gravidade dos diferentes quadros clínicos. Por exemplo, dos dados mostrados na Tabela 5, podemos afirmar que, o grau de gravidade do quadro clínico coma $(E M=380,00)$ é aproximadamente quatro vezes maior que o grau de gravidade do quadro clínico esgotamento nervoso $(\mathrm{EM}=95,56)$, ou aproximadamente vinte vezes maior que o grau de gravidade do quadro clínico período pré-menstrual $(\mathrm{EM}=18,90)$.

\section{COMPARISON BETWEEN THE PSYCHOPHYSICAL METHODS OF MAGNITUDE AND CATEGORIES ESTIMATION OF ILLNESS SERIOUSNESS}

In this study, 15 diagnoses were selected and evaluated by the methods of estimation of magnitudes and estimation of categories. These methods were performed by 46 subjects (10 physicians, 20 nurses and 16 psychologists). The results show that the non-metric continuum of seriousness of an illness exhibits prothetic characteristics and the variability of estimates is a linear function of the magnitude estimation, thus confirming that Ekman's law is also valid for non-metric continua.

KEY WORDS: psychophysics

\section{COMPARACIÓN ENTRE LOS MÉTODOS PSICOFÍSICOS ESCALARES DE ESTIMACIÓN DE MAGNITUDES Y DE ESTIMACIÓN DE CATEGORÍAS DE LA GRAVEDAD DE CUADROS CLÍNICOS}

En este estudio, 15 diagnósticos, fueron seleccionados y evaluados por el método de estimación de magnitud y de categoría. Los instrumentos fueron aplicados a 46 participantes (20 enfermeros, 16 psicólogos y 10 médicos). Los resultados indican que la escala psicofísica de los cuadros clínicos es un continuo protético, además de la confirmación de la validez de la Ley de Ekman también para continuos no métricos. 


\section{REFERÊNCIAS BIBLIOGRÁFICAS}

01. KAMIZAKI, R.; SOUSA, F.A.E.F.; SANT'ANA, R.P.M., SILVA, J.A. Estimação de magnitude da gravidade de quadros clínicos = um enfoque da psicofísica clínica. Rev.latino-am.enfermagem, Ribeirão Preto, v. 7, n. 2, p. 55-62, 1999.

02. STEVENS, S.S. A metric for the social consensus. Science, v. 151, p. 530-541, 1966.

03. STEVENS, S.S. Psychophysics: introduction to its perceptual, neural and social prospects. In: STEVENS, G. (ed.). New York: Wiley, 1975.
04. VOLICER, B.J.; BOHANNON, M.W. A hospital stress rating scale. Nursing Research, v. 24, p. 352-364, 1975.

05. WYLER, A.R.; MASUDA, M.; HOLMES, T.H. Seriousness of illness rating scale. Journal of Psychosomatic Research, v. 11, p. 363-374, 1968.

06. WYLER, A. R.; MASUDA, M.; HOLMES, T. H. The seriousness of illness rating scale: Reproducibility. Journal of Psychosomatic Research, v. 14, p. 59-64, 1970.

07. WYLER, A.R.; MASUDA, M.; HOLMES, T.H. Magnitude of life events and seriousness of illness. Psychosomatic Medicine, v. 33, p. 115122, 1971. 